\title{
Knockdown of FAK inhibits the invasion and metastasis of Tca-8113 cells in vitro
}

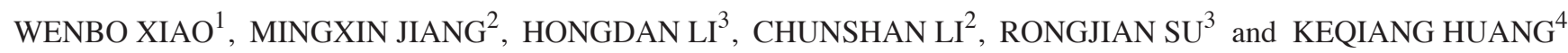 \\ ${ }^{1}$ Liaoning Medical College Graduate School; ${ }^{2}$ The Second Affiliated Hospital of Liaoning Medical College; \\ ${ }^{3}$ Key Lab of Molecular and Cellular Biology, Department of Education of Liaoning Province, \\ Central Laboratory of Liaoning Medical College; ${ }^{4}$ The Oral Department of Liaoning Medical College, \\ Jinzhou, Liaoning 121000, P.R. China
}

Received February 1, 2013; Accepted June 17, 2013

DOI: $10.3892 / \mathrm{mmr} .2013 .1555$

\begin{abstract}
Tongue cancer originating on the surface of the tongue is most commonly squamous cell carcinoma, which has a higher invasive ability and a lower survival rate compared with other forms of tongue cancer. Notably, tongue squamous cell carcinomas metastasize into lymph nodes at early stages. Focal adhesion kinase (FAK) is an important protein tyrosine kinase involved in invasion and metastasis of cancer cells. In the present study, the role of FAK in the invasion and metastasis of tongue cancer was evaluated and the underlying mechanisms involved in this process were explored. FAK knockdown was performed using shRNA in the tongue cancer cell line, Tca-8113, and the invasion and metastasis potentials were analyzed using wound healing and transwell assays, respectively. Cytoskeletal arrangement was detected by fluorescence using TRITC-conjugated phalloidin staining. The activity of matrix metalloproteinase (MMP)-2 and -9 was examined by gelatin zymography. Paxillin distribution was observed by immunofluorescence. The levels of E-cadherin, N-cadherin, MMP-2 and -9, and c-Jun N-terminal kinase (JNK) was detected by western blot analysis. Wound healing and transwell assays demonstrated that FAK knockdown inhibited the invasion and metastasis of Tca-8113 cells. Further analysis revealed that FAK knockdown caused the rearrangement of the cytoskeleton and decreased the activity of MMP-2 and -9. Immunofluorescence analysis revealed that downregulation of FAK induced the relocalization of paxillin. Paxillin accumulated as dots and patches at the cell membrane in control cells. By contrast, in FAK knockdown cells, paxillin was distributed homogeneously in the cytoplasm. Western blot analysis revealed that FAK knockdown inhibited epithelialmesenchymal transition (EMT) and decreased levels of
\end{abstract}

Correspondence to: Professor Keqiang Huang, The Oral Department of Liaoning Medical College, 40 Songpo Road, Jinzhou, Liaoning 121000, P.R. China

E-mail: hkq9@163.com

Key words: FAK, invasion, metastasis, Tca-8113
MMP-2 and -9, and p-JNK. Knockdown of FAK inhibits the invasion and metastasis of Tca- 8113 by decreasing MMP-2 and -9 activities and led to the rearrangement of the cytoskeleton and inhibited the EMT.

\section{Introduction}

Tongue cancer is a form of oral cancer. The majority of cases of tongue cancer are squamous cell carcinomas which originate from the surface layer of the tongue. At present, tongue squamous cell carcinoma is the most common form of oral cancer and is associated with early lymph node metastasis and poor survival. Early tongue cancer is often treatable with surgery or radiation therapy, depending upon the location and size. Therefore, a growing emphasis has recently been placed on inhibiting the invasion and metastasis of tongue cancer. However, the effect of inhibition remains unclear.

Focal adhesion kinase (FAK) (1) functions as a phosphorylation-regulated signaling scaffold and is important for adhesion turnover, Rho-family GTPase activation, cell migration and cross-talk between growth-factor signalling and integrins (Mitra et al., 2005) (2). This ubiquitously expressed, essential protein contains an N-terminal FERM domain, a central kinase domain, proline-rich regions and a C-terminal focal-adhesion-targeting domain that interacts with paxillin (3). p130Cas-Crk signaling is regulated by interacting proteins. Cells utilize FAK-Src activity to recruit a p130Cas-Crk complex that promotes Rac GTPase activity, leading to lamellipodia formation in migrating cells (4-6). Rates of focal adhesion formation/turnover are affected by FAK-Src complex phosphorylation of paxillin, paxillin kinase linker and p130Cas. Elevated extracellular signal regulated kinase 2 and c-Jun N-terminal kinase (JNK) promote increased gene transcription of target proteins leading to enhanced secretion of matrix metalloproteinase (MMP)-2 and -9 . Protease localization and activation at the leading edge of migrating tumor cells is regulated by integrins and facilitates matrix degradation. However, the mechanism of FAK in invasion and metastasis of tongue cancer remains unclear.

In the present study, Tca- 8113 cells were selected to analyze the effect of FAK knockdown on tongue cancer cells, demon- 
strating that FAK promotes Tca-8113 invasion and metastasis by JNK signaling.

\section{Materials and methods}

Cell culture. Human tongue cancer TCA-8113 cells were purchased from the Cell Bank of Type Culture Collection of the Chinese Academy of Sciences (Shanghai, China). The cells were cultured in complete DMEM supplemented with $10 \%$ fetal bovine serum, $2 \mathrm{mM}$ glutamine, $100 \mathrm{U} / \mathrm{ml}$ penicillin and $100 \mu \mathrm{g} / \mathrm{ml}$ streptomycin at $37^{\circ} \mathrm{C}$, in a $5 \% \mathrm{CO}_{2}-95 \% \mathrm{O}_{2}$ atmosphere and passaged every 3-5 days. The study was approved by the ethics committee of Liaoning medical college, Jinzhou city, China.

Knockdown of FAK. shRNAs against FAK were designed and synthesized by Shanghai Genechem Co., Ltd. (Shanghai, China). The sequence of the sense strand was as follows: 5'-GCTAGTGACGTATGGATGT-3'. TCA-8113 cells in exponential growth phase were plated in six-well plates and allowed to adhere for $24 \mathrm{~h}$ prior to transfection. Transfection was performed using Lipofectamine 2000 according to the manufacturer's instructions (Invitrogen Life Technologies, Carlsbad, CA, USA). Briefly, cells were cultured to $80 \%$ confluence in a six-well culture plate and transfected at a 1:4 ratio (plasmid DNA:transfection reagent $=5 \mu \mathrm{g}: 20 \mu \mathrm{l}$ ) (7). Control cells were transfected with control RNA under the same conditions. Transfection efficiency was analyzed by western blot analysis at $72 \mathrm{~h}$.

Transwell assay. The transwell assay was performed using 24-well transwells (Costar, Cambridge, MA, USA). Following $48 \mathrm{~h}$ transfection, cells were seeded into fibronectin gel pre-coated, porous upper chamber inserts $\left(1 \times 10^{4}\right.$ cells/well $)$ and allowed to invade for $24 \mathrm{~h}$. Following $24 \mathrm{~h}$, inserts were inverted and stained with Hoechst 33258. The number of invaded cells were observed and quantified using a Zeiss Axio Scope A1 fluorescent microscope (Carl Zeiss AG, Oberkochen, Germany; magnification, x100). Five fields were randomly selected and the number of penetrated cells was quantified.

Wound healing assay. Following $48 \mathrm{~h}$ transfection, cells were harvested and seeded in six-well plates $\left(1 \times 10^{5}\right.$ cells/well $)$ and cultured until cells reached. The cells were scratched using a yellow pipette tip, washed with PBS three times and cultured in serum-free medium for $24 \mathrm{~h}$. The status of cell migration was represented by the rate of wound closure.

Western blot analysis. Following 48 h siRNA transfection, cells were harvested, washed twice with ice-cold PBS, then lysed in RIPA buffer [150 mM NaCl, 1\% NP-40, $1 \%$ SDS, $1 \mathrm{mM}$ PMSF, $10 \mu \mathrm{g} / \mathrm{ml}$ leupeptin, $1 \mathrm{mM}$ aprotinin and $50 \mathrm{mM}$ Tris- $\mathrm{Cl}$ (pH 7.4)]. The cell lysate was cleared by centrifugation at $12,000 \mathrm{x} \mathrm{g}$ for $15 \mathrm{~min}$. Cell lysate containing $50 \mu \mathrm{g}$ protein was separated by $10 \%$ SDS-PAGE and the protein was transferred onto polyvinylidene fluoride membranes. Following blocking with 5\% non-fat dry milk in Tris-buffered saline, the membrane was incubated overnight with primary antibodies against FAK (FAK sampler kit, 9330; Cell Signaling, Danvers,
MA, USA)., MMP-2 (4022; Cell Signaling), MMP-9 (3852; Cell Signaling), MMP-14 (Ab51074; Abcam, Cambridge, MA, USA), N-cadherin (sc-59987; Santa Cruz Biotechnology, Santa Cruz, CA, USA), E-cadherin (sc-52328; Santa Cruz), Paxillin (2542; Cell Signaling), p-Tyr118 (2541; Cell Signaling) and p-S178 (ab51617; Abcam). Following this, the membrane was incubated with appropriate secondary antibodies for $1 \mathrm{~h}$ and stained with ECL. Protein expression levels of the targeted proteins were analyzed by UVP gel analysis system and images of the membranes were captured (7).

Immunofluorescence. Following 48 h siRNA transfection, cells were harvested and replated on fibronectin pre-coated coverslips in 6-well plates. After $24 \mathrm{~h}$, cells were fixed in $3.7 \%$ formaldehyde in PBS, permeabilized with $0.1 \%$ Triton X-100 in PBS, blocked with PBS containing 1\% BSA and incubated with specific primary antibodies for $60 \mathrm{~min}$. Next, the slides were mounted by $95 \%$ glycerol and observed using the Zeiss A-1 fluorescent microscope (Carl Zeiss AG).

Cytoskeleton staining. Cells were harvested and replated on fibronectin-coated coverslips $(10 \mu \mathrm{g} / \mathrm{ml})$. Following $24 \mathrm{~h}$, cells were fixed in $3.7 \%$ formaldehyde in PBS, permeabilized with $0.1 \%$ Triton X-100 in PBS, blocked with PBS containing $1 \%$ BSA and incubated with TRITC-conjugated phalloidin (Sigma-Aldrich, St. Louis, MO, USA) for $30 \mathrm{~min}$. The slides were mounted by $95 \%$ glycerol and observed using the Zeiss Axio Scope A1 fluorescent microscope.

Gelatin zymography. Conditioned medium was collected and concentrated by 2 -fold using a centrifugal concentrator. Equal amounts of protein were loaded and separated by $10 \%$ polyacrylamide gel containing $1 \mathrm{~g} / 1$ gelatin. The gels were re-natured in $2.5 \%$ Triton-X-100 with gentle agitation for $30 \mathrm{~min}$ at room temperature. The gel was pretreated with developing buffer [ $5 \mathrm{mM} \mathrm{CaCl}_{2}, 50 \mathrm{mM}$ Tris, $0.2 \mathrm{mM} \mathrm{NaCl}$ and $0.02 \%$ Brij-35 ( $\mathrm{pH} \mathrm{7.5)]} \mathrm{for} 30 \mathrm{~min}$ at room temperature, then developed in developing buffer overnight at $37^{\circ} \mathrm{C}$. Following this, the gel was stained with Coomassie Brilliant Blue R-250 for 30 min and destained with destaining solution. Protease activity was analyzed using a gel imaging and analysis system $(9,10)$.

Statistical analysis. SPSS 13.0 statistical software was used for analysis of the data (SPSS, Inc., Chicago, IL, USA). Data are expressed as the mean $\pm \mathrm{SD}$. $\mathrm{P}<0.05$ was considered to indicate a statistically significant difference.

\section{Results}

Knockdown of FAK inhibits the invasion and metastasis of TCA-8113. To determine whether FAK knockdown affects the invasion and metastasis of TCA-8113, FAK knockdown was performed by transfection of FAK-specific shRNA and the effect of RNA interference was determined using western blot analysis (Fig. 1C). The invasion and metastasis potential of FAK knockdown cells was analyzed further by wound healing and transwell assays (11). Wound healing assay revealed that the metastasis of FAK knockdown cells was significantly decreased $(-50 \%)$ compared with the control 

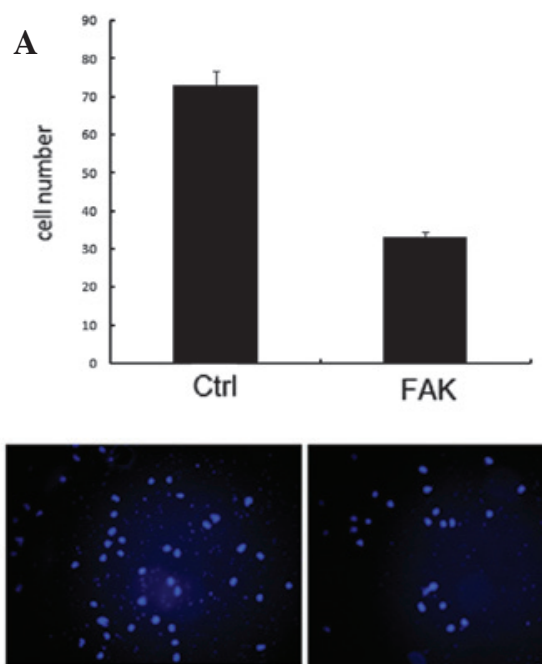

Ctrl

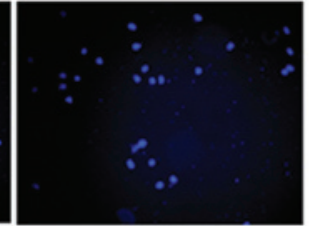

FAK
B
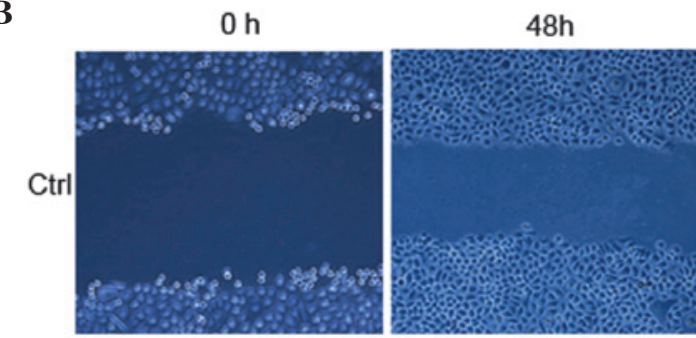

C

Ctrl

FAK

FAK

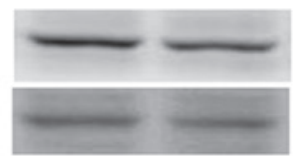

FAK
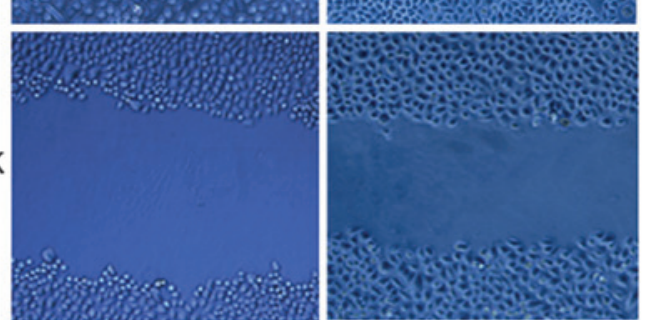

$\mathrm{N}-\mathrm{Cad}$

E-Cad

$\beta$-actin

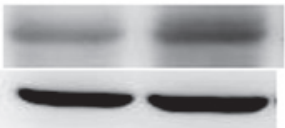

Figure 1. Downregulation of FAK inhibits EMT. (A) Transwell assay demonstrates that downregulation of FAK expression decreases cell invasion. Upper panel, analysis of Ctrl and FAK downregulated cells and lower panel, invaded cells in the two groups (magnification, x100). (B) Wound healing assay demonstrates that downregulation of FAK inhibits cell invasion at $48 \mathrm{~h}$. (C) Western blot analysis indicates that knockdown of FAK increases the expression of E-cad and decreases the expression of $\mathrm{N}$-cad. Ctrl, control; Cad, cadherin; FAK, focal adhesion kinase; EMT, epithelial-mesenchymal transition.

A

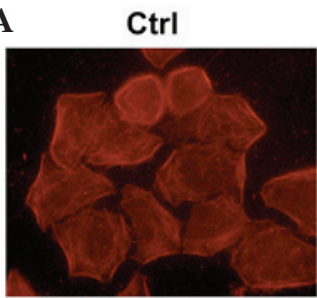

FAK

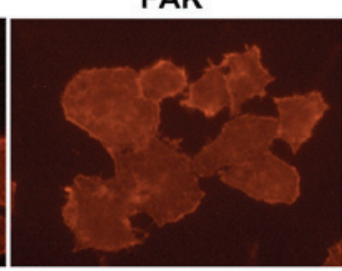

B

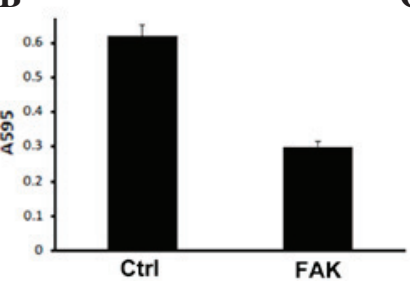

C

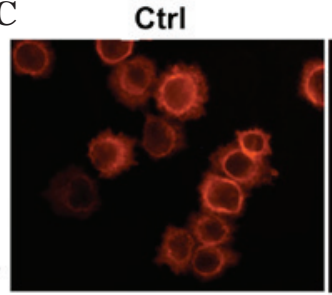

FAK

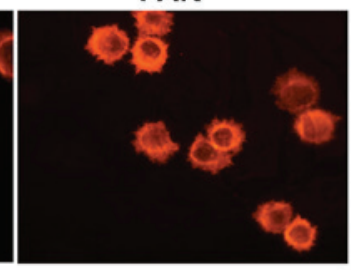

Figure 2. Knockdown of FAK leads to cytoskeletal rearrangement and inhibition of cell adhesion and spreading. (A) Immunofluorescence reveals that FAK knockdown inhibits stress fiber formation. (B) Cell adhesion assay demonstrates that Ctrl cell adherence was higher than knockdown of FAK cells. (C) Cell spreading assay revealed that Ctrl cells exhibit improved spread compared with knockdown of FAK cells. Knockdown of FAK reduced the activity of MMP-2 and -9. Ctrl, control; FAK, focal adhesion kinase; MMP, matrix metalloproteinase.
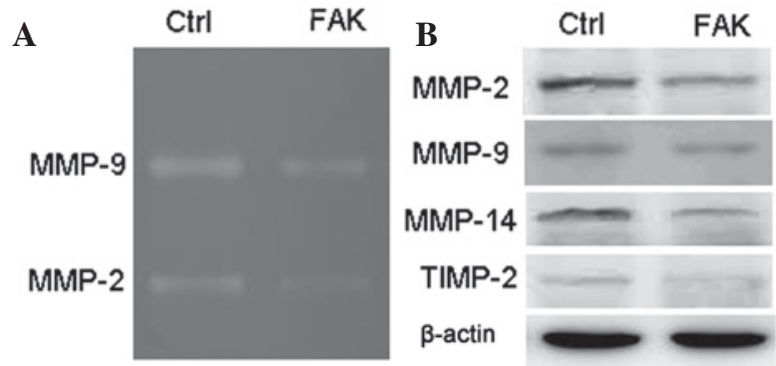

Figure 3. Knockdown of FAK reduces the activity of MMP-2 and -9 . (A) Gelatin zymography demonstrates that knockdown of FAK inhibits the activity of MMP-2 and -9. (B) Western blot analysis reveals that knockdown of FAK decreases the expression of MMP-2, -9 and -14, and TIMP-2. Ctrl, control; FAK, focal adhesion kinase.

cells (Fig. 1B). Transwell assay demonstrated that knockdown of FAK in TCA-8113 cells reduced the invasion potential to $46 \%$ compared with control cells (Fig. 1A).

Downregulation of FAK in TCA-8113 inhibits epithelialmesenchymal transition (EMT). EMT is essential for the invasion and metastasis of TCA-8113 (12). To elucidate the

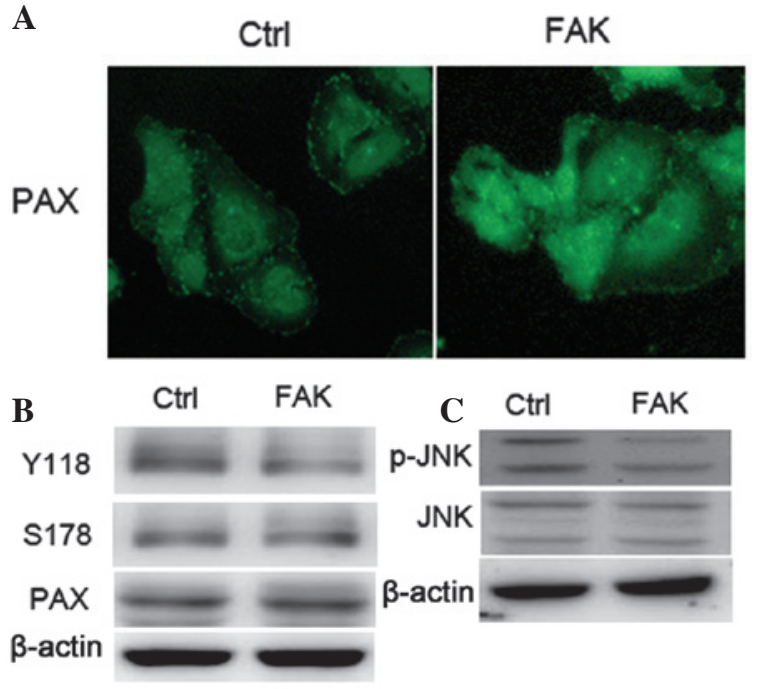

Figure 4. Knockdown of FAK initiates pax redistribution and JNK downregulation. (A) Immunofluorescence demonstrates that inhibition of FAK initiates paxillin distribution changes from the cell membrane (Ctrl) to the cytoplasm (FAK). (B) Western blot analysis demonstrates that inhibition of FAK downregulates the expression of total pax and pax Y118 and S178. (C) Western blot analysis reveals that inhibition of FAK decreases p-JNK, but does not affect total JNK. $\beta$-actin was used as an internal control. FAK, focal adhesion kinase; pax, paxillin; JNK, c-Jun N-terminal kinase. 
underlying mechanisms, the effects of FAK knockdown on EMT were examined by analyzing the expression of E-cadherin and N-cadherin in FAK knockdown cells. Knockdown of FAK increased the levels of E-cadherin, whereas the expression of $\mathrm{N}$-cadherin was reduced, indicating that FAK knockdown inhibited EMT.

Knockdown of FAK leads to cytoskeletal rearrangement and inhibition of cell adhesion and spreading. To determine the role of FAK in the regulation of cytoskeletal dynamics, cytoskeletal dynamics (13) were analyzed by TRITC-conjugated phalloidin staining (Fig. 2A). Actin filaments were distributed mainly on the periphery and no marked stress fiber formation was observed in the cell cortex in FAK knockdown cells, indicating that knockdown of FAK inhibits stress fiber formation. The status of cell adhesion and spreading was analyzed further in FAK knockdown cells using cell adhesion and spreading assays (Fig. 2B). The cell adhesion assay revealed that knockdown of FAK inhibited cell adhesion to fibronectin-coated substrate. In addition, the cell spreading assay demonstrated that knockdown of FAK reduced the spreading of tumor cells.

Knockdown of FAK induces paxillin redistribution and inhibits the activity of JNK. To identify whether FAK knockdown affects extracellular matrix degradation (14), the activity of MMP-2 and -9 was determined by gelatin zymography in FAK knockdown cells (Fig. 3A). Knockdown of FAK decreased the activity of MMP-2 and -9. In addition, the expression of several MMP family members, including MMP-2, -9 and -14, and TIMP-2, was determined in FAK knockdown cells. FAK knockdown decreased the levels of MMP-2, -9 and -14 , and TIMP-2 (Fig. 3B).

Paxillin is an important signal transduction adaptor protein involved in the regulation of tumor invasion and metastasis (15). The distribution of paxillin in FAK knockdown cells was analyzed and paxillin was observed to be distributed homogeneously in the cytoplasm in FAK knockdown cells. However, paxillin was found to accumulate at the cell membrane in control cells. In addition, the phosphorylation levels of paxillin at residues Y118 and S178 were analyzed by western blot analysis (16). Western blot analysis revealed that the phosphorylation levels of Y118 and S178 were significantly decreased (Fig. 4).

JNK is a well-known Ser and Thr protein kinase. Therefore, JNK activity was analyzed in FAK knockdown cells by western blot analysis. The results revealed that the phosphorylation level of JNK was significantly decreased in FAK knockdown cells as compared with control cells, indicating that FAK knockdown inhibited the activity of JNK. However, the expression of JNK was comparable in FAK knockdown and control cells.

\section{Discussion}

Due to its characteristic feature of early lymph node metastasis, tongue cancer is difficult to treat. Of note, surgical resection has been found to correlate with tissue and organ dysfunction leading to a poorer quality of life for patients $(17,18)$. Therefore, the identification of novel therapeutic target molecules to inhibit invasion and metastasis in tongue cancer is vital. At present, FAK is known to be overexpressed in various forms of cancer $(19,20)$. A number of studies have demonstrated that FAK is useful as a molecular marker of nodal metastasis in cancer cells (21-23). In the present study, shFAK was used to treat Tca- 8113 cells to determine the mechanisms associated with invasion and metastasis of tongue cancer.

Firstly, western blot analysis was used to analyze the knockdown efficiency of shFAK in Tca-8113. Next, the invasive abilities of shFAK-treated and control Tca-8113 cells was determined by wound healing assay. shFAK was found to inhibit the invasion of Tca-8113 cells. To further validate this result, the activity of a number of MMPs was analyzed by western blot analysis. MMPs are important for tumor invasion and metastasis, and among numerous MMPs, MMP-2 and -9 are well characterized in invasion and metastasis (24). Western blot analysis revealed that shFAK decreases the expression of MMP-2, -9 and -14 in cells. In addition, knockdown of FAK was found to decrease the expression of TIMP-2.

To elucidate the effect of shFAK on the cytoskeleton $(25,26)$, shFAK-treated TCA-8113 cells were compared with control cells. The results indicate that shFAK inhibits stress fiber formation and results in rearrangement of the cytoskeleton. In addition, shFAK was found to initiate paxillin redistribution and promote changes in paxillin expression from the cell membrane to the cytoplasm. These observations indicate that paxillin functions as an anchor for the FAK-Src complex and activates downstream signaling (27-29). Downregulated FAK promotes paxillin redistribution. Western blot analysis also revealed that inhibition of FAK downregulates paxillin expression and phosphorylation.

To elucidate the mechanism by which shFAK inhibits the invasion and metastasis of tongue cancer cells, the expression of JNK was analyzed as JNK is known to regulate MMP-2 and -9. shFAK was found to inhibit the activity of JNK. As JNK cannot activate c-jun, which is downstream of JNK, the expression of MMP-2/9 and MMP-14 was downregulated.

In summary, the results of the current study indicate that the downregulation of FAK inhibits Tca-8113 invasion and metastasis by JNK and c-Jun signaling. Elevated JNK promotes increased gene transcription of target proteins leading to enhanced secretion of MMP-2, -9 and -14 (30). Following this, the leading edge of migrating tumor cells is activated, cytoskeletal rearrangement occurs and invadopodia formation is enhanced. The inhibition of FAK leads to the reduction of JNK phosphorylation, inhibiting c-jun activity, which is an important TF in the nucleus. The reduction of $\mathrm{c}$-jun inhibits MMP-2 activity, as well as decreasing cell invasion

As shFAK inhibits the invasion and metastasis of tongue cancer cells, the molecular pathway may provide a potential therapeutic target for tongue tumor.

\section{References}

1. Schaller MD, Borgan CA, Cobb BS, et al: Focal adhesion kinase and associated proteins. Curr Opin Cell Biol 6: 705-710,1994

2. Mitra SK, Hanson DA and Schlaepfer DD: Focal adhesion kinase: in command and control of cell motility. Nat Rev Mol Cell Biol 6: 56-68, 2005.

3. Jelena P and Derrick B: Paxillin phosphorylation and complexing with Erk and FAK are regulated by PLD activity in MDA-MB-231 cells. Cell Signal 24: 1531-1540, 2012. 
4. Abdelkader H, Maya B, Monique D, et al: Regulation of focal adhesion dynamics and disassembly by phosphorylation of FAK at tyrosine 397. J Cell Sci 118: 4415-4425, 2005.

5. Vita MG, Sheila F, Baotran TH, et al: A small molecule focal adhesion kinase (FAK) inhibitor, targeting Y397 site: 1-(2-hydroxyethyl)-3, 5, 7-triaza-1-azoniatricyclo [3.3.1.1(3,7)] decane; bromide effectively inhibits FAK autophosphorylation activity and decreases cancer cell viability, clonogenicity and tumor growth in vivo. Carcinogenesis 33: 1004-1013, 2012.

6. Tanja P, Virginia E, Kai K, et al: Sulfur mustard induces differentiation in human primary keratinocytes: Opposite roles of p38 and ERK1/2 MAPK. Toxicol Lett 204: 43-51, 2011.

7. Hochwald SN, Nyberg C, Zheng M, et al: A novel small molecule inhibitor of FAK decreases growth of human pancreatic cancer. Cell Cycle 8: 2435-2443, 2009.

8. Berger S, Dyugovskaya L, Polyakov A and Lavie L : Short-term fibronectin treatment induces endothelial-like and angiogenic properties in monocyte-derived immature dendritic cells: Involvement of intracellular VEGF and MAPK regulation. Eur J Cell Biol 91: 640-653, 2012.

9. Tanjoni I, Walsh C, Uryu S, et al: PND-1186 FAK inhibitor selectively promotes tumor cell apoptosis in three-dimensional environments. Cancer Biol Ther 9: 764-777, 2010.

10. Beierle EA, Ma X, Stewart J, et al: Inhibition of focal adhesion kinase decreases tumor growth in human neuroblastoma. Cell Cycle 9: 1005-1015, 2010.

11. Broughton G 2nd, Janis JE and Attinger CE: The basic science of wound healing. Plast Reconstr Surg 117 (7 Suppl): 12S-34S, 2006.

12. CJ Creighton, JC Chang, JM Rosen, et al: A Gene Expression Signature Associated with "K-Ras Addiction" Reveals Regulators of EMT and Tumor Cell Survival. Cancer Cell 15: 489-500, 2009.

13. P Bannasch, H Zerban and D Mayer: The cytoskeleton in tumor cells. Pathology 175: 196-211, 1982

14. Stetler-Stevenson WG, Aznavoorian S and Liotta LA: Tumor cell interactions with the extracellular matrix during invasion and metastasis. Annu Rev Cell Biol 9: 541-573, 1993.

15. Turner C E: Paxillin and focal adhesion signalling. Nat Cell Biol 2: E231-E236, 2000

16. Chen J and Gallo KA: MLK3 Regulates Paxillin Phosphorylation in Chemokine-Mediated Breast Cancer Cell Migration and Invasion to Drive Metastasis. Cancer Res 72: 4130-4140, 2012.

17. Ludmila TT, Plamen IP, Daniel K, et al: Endosonographic assessment of rectal cancer after neoadjuvant radiotherapy. Med Ultrason 14: 19-23, 2012.

18. Tankova L, Stoilov G, Kovatchki D, et al: Comparative evaluation of angiogenesis in rectal cancer using Doppler ultrasound and immunohistochemical assessment. Compt Rend Acad Bulg Sci 63: 163-166, 2010.
19. Sudakoff GS, Gasparaitis A, Michelassi F, et al: Endorectal color Doppler imaging of primary and recurrent rectal wall tumours: Preliminary experience. AJR Am J Roentgenol 166: 55-61, 1996.

20. Xiaoban X, Lijun Z, Caleb M, Reyes, et al: APPL1 mediates adiponectin-stimulated $\mathrm{p} 38$ MAPK activation by scaffolding the TAK1-MKK3-p38 MAPK pathway. Am J Physiol Endocrinol Metab 300:E103-110, 2011.

21. Ito R, Oue N, Zhu X, et al: Expression of integrin-linked kinase is closely correlated with invasion and metastasis of gastric carcinoma. Virchows Arch 442: 118-123, 2003.

22. Owens LV, Xu L, Dent GA, Yang X, et al: Focal adhesion kinase as a marker of invasive potential in differentiated human thyroid cancer. Ann Surg Oncol 3: 100-105, 1996.

23. Miyazaki T, Kato H, Nakajima M, et al: FAK overexpression is correlated with tumour invasiveness and lymph node metastasis in oesophageal squamous cell carcinoma. Br J Cancer 89: 140-145, 2003.

24. Masson V, de la Ballina LR, Munaut C, et al: Contribution of host MMP-2 and MMP-9 to promote tumor vascularization and invasion of malignant keratinocytes. FASEB J 19: 234-236, 2005.

25. Zhou L, Deepa SS, Etzler JC, et al: Adiponectin activates AMP-activated protein kinase in muscle cells via APPL1/ LKB1-dependent and phospholipase C/Ca2_/Ca2_/calmodulin-dependent protein kinase kinase-dependent pathways. J Biol Chem 284: 22426-22435, 2009.

26. Heneghan JP, Salem RR, Lange RC, et al: Transrectal sonography in staging rectal carcinoma: The role of gray-scale, color-flow and Doppler imaging analysis. AJR Am J Roentgenol 169: 1247-1252, 1997.

27. Liu M, Wilk SA, Wang A, et al: Resveratrol inhibits mTOR signaling by promoting the interaction between mTOR and DEPTOR. J Biol Chem 285: 36387-36394, 2010.

28. Schaller MD: Paxillin: a focal adhesion-associated adaptor protein. Oncogene 20: 6459-6472, 2001.

29. Bae GU, Lee JR, Kim BG, et al: Cdo interacts with APPL1 and activates AKT in myoblast differentiation. Mol Biol Cell 21: 2399-2411, 2010

30. Cheung LW, Leung PC, Wong AS: Gonadotropin-Releasing Hormone Promotes Ovarian Cancer Cell Invasiveness through c-Jun NH2-Terminal Kinase-Mediated Activation of Matrix Metalloproteinase (MMP)-2 and MMP-9. Cancer Res 66: 10902-10910, 2006. 